\title{
Characterization of CD44-positive Cancer Stem-like Cells in COLO 201 Cells
}

\author{
HIROHISA OKUYAMA ${ }^{1,2}$, WATARU NOGAMI ${ }^{2}$, YUJI SATO ${ }^{2}$, \\ HIROSHI YOSHIDA ${ }^{2}$, YUMI TONA ${ }^{2}$ and YOSHITAKA TANAKA ${ }^{1}$ \\ ${ }^{1}$ Division of Pharmaceutical Cell Biology, Graduate School of \\ Pharmaceutical Sciences, Kyushu University, Fukuoka, Japan; \\ ${ }^{2}$ Drug Discovery \& Disease Research Laboratory, Shionogi \& Co., Ltd., Osaka, Japan
}

\begin{abstract}
Background/Aim: Cancer stem cells (CSCs) are considered to be one of the causes of tumor recurrence after chemotherapy. The purpose of our study was to isolate CSCs from human colorectal cancer cell (CRC) lines. Materials and Methods: Nine CRC lines were screened based on the expression level of potential CSC markers to identify putative CSCs. Tumor formation capacity in immunodeficient mice was compared with that of their counterparts. Stemness, differentiation potency and sensitivity to 5-fluorouracil (5$F U)$, in vitro, were also assessed. Microarray analysis was used to characterize the features of the putative CSCs. Results: COLO 201 cells were separated into two populations based on CD44 expression. CD44 positive $\left(C D 44^{+}\right)$cells showed significantly higher tumor formation capacity than $\mathrm{CD}_{44^{-}}$cells in immunodeficient mice. $\mathrm{CD} 44^{+}$ cells also possessed stemness properties and lower sensitivity to 5-FU in vitro. Moreover, cancer stemness and chemoresistance-related genes were highly up-regulated in CD44 ${ }^{+}$cells. Conclusion: $\mathrm{CD} 44^{+}$COLO 201 cells possessed the features of CSCs; therefore, the present CSC model could serve as a valuable tool to accelerate CSC research.
\end{abstract}

Colorectal cancer (CRC) is one of the most commonly diagnosed cancers worldwide. CRC remains the second leading cause of cancer-related deaths in the world, and tumor recurrence after chemotherapy is a serious concern (1). Increasing evidence indicates the existence of a subpopulation of tumor cells, termed cancer stem cells (CSCs), which have the ability of self-renewal and

Correspondence to: Hirohisa Okuyama, Drug Discovery \& Disease Research Laboratory, Shionogi \& Co., Ltd, Osaka, 561-0825, Japan. Tel: +816331 7238, e-mail: hirohisa.okuyama@shionogi.co.jp

Key Words: Cancer stem cells, colorectal cancer, stemness, COLO 201 cells, CD44-positive cancer cells. differentiation, and seem to be responsible for initiating and sustaining tumors (2). They are reported to be more resistant to chemotherapy, radiotherapy and immunotherapy than nonCSCs $(3,4)$. Therefore, CSCs may be a cause of recurrence following tumor regression after such treatments (5).

Researchers have isolated CSCs by exploring multiple cell surface markers in a variety of tumors (6). Regarding CRC, CSCs have been identified in primary tumors by their expression of CD44 (7), CD133 (8), CD166 (7) or LGR5 (9). Although functional molecules and pathways have been identified to isolate them using these cell surface markers, therapeutic approaches toward targeting them have not yet been successful (10). In clinical settings, it is difficult to stably obtain sufficient primary CSCs and to maintain them, as there are differences in the expression of CSC markers and the presence of these cells among patients (11). Therefore, the identification of CSCs in cancer cell lines could provide a useful tool for the development of therapies that target CSCs.

In this study, we investigated cancer stemness properties using validated cell lines newly purchased from the American Type Culture Collection (ATCC) because different methods for passaging cell lines in different laboratories could lead to changes in cell characteristics. We found that COLO 201 cells could be separated into two populations based on CD44 expression, and CD44 ${ }^{+}$cells were found to possess the features of CSCs. These results suggest that the development of a therapeutic approach targeting $\mathrm{CD} 44^{+}$ COLO 201 cells might contribute to effective treatment against CSCs in clinical situations.

\section{Materials and Methods}

Cell cultures and reagents. Human colon carcinoma cell lines (NCIH747, SW48, WiDr, LS180, LS411N, HT-29, COLO 201, COLO 320DM, HCT15) were purchased from the American Type Culture Collection (Manassas, VA, USA) and used within 10 passages. Cell lines were maintained in DMEM (Wako, Osaka, Japan) supplemented 
with $10 \%$ fetal bovine serum (Hyclone, Logan, UT, USA), 100 units $/ \mathrm{ml}$ penicillin, $100 \mu \mathrm{g} / \mathrm{ml}$ streptomycin and $25 \mu \mathrm{g} / \mathrm{ml}$ amphotericin B (GIBCO, Thermo Fisher Scientific, Waltham, MA, USA) To assess the effect of sphere culture, cells were cultured in Knockout DMEM/F-12 (Thermo Fisher Scientific) with B27 without Vitamin A (Thermo Fisher Scientific), $20 \mathrm{ng} / \mathrm{ml}$ EGF (Peprotech, Rocky Hill, NJ, USA), $20 \mathrm{ng} / \mathrm{ml} \mathrm{bFGF} \mathrm{(Peprotech)} \mathrm{and} 2 \mu \mathrm{g} / \mathrm{ml}$ heparin (STEMCELL, Vancouver, Canada) on an ultra-low attachment plate. All cultures were maintained at $37^{\circ} \mathrm{C}$ under $5 \% \mathrm{CO}_{2}$.

Flow cytometric analysis and cell sorting. Cells were incubated with fluorescence-labeled monoclonal antibodies at $4{ }^{\circ} \mathrm{C}$ for $35 \mathrm{~min}$ in the dark. The following monoclonal antibodies were used: PE- or APClabeled anti-CD44 (clone G44-26; BD Biosciences, San Diego, USA), PE-labeled anti-CD133 (clone AC133; Miltenyi Biotec, Bergisch Gladbach, Germany), PE-labeled anti-CD166 (clone 3A6; Biolegend, San Diego, CA, USA). PE- or APC-mouse IgG2b (clone 27-35; BD Biosciences) and PE mouse IgG1 (clone IS5-21F5; Miltenyi Biotec/clone MOPC-21; BD Biosciences) were used as isotype controls. At least $1 \times 10^{4}$ cells were analyzed for each experiment. Flow cytometry was performed using a MACSQuant Analyzer 10 (Miltenyi Biotec) and cell sorting was performed using a FACSAria ${ }^{\mathrm{TM}}$ II (BD Biosciences). Self-renewal and differentiation analyses were based on the expression of CD44. A single CD44+ or CD44- cell sorted from COLO 201 was cultured in each well of a 96-well plate under normal conditions for 3 weeks. Cells in each well were stained with APC-labeled anti-CD44 antibody and analyzed by MACSQuant.

Tumorigenicity assay in vivo. Unsorted or sorted cells based on the expression of CD44 were suspended in a 1:1 mixture of DMEM and growth factor-reduced Matrigel matrix (BD Biosciences); a total volume of $50 \mu \mathrm{l}$ was injected subcutaneously into the flank of each anesthetized recipient mouse. Tumor volume measurement was performed two or three times a week with a digital caliper. Tumor volumes were calculated according to the formula (length $\times$ width $^{2}$ )/2. Nude mice or NOD/SCID mice were obtained from CLEA Japan. All procedures were approved by the Animal Care and Use Committee of Shionogi \& Co., Ltd. All experiments were performed in accordance with the Committee's guidelines.

Immunohistochemistry. Formalin-fixed paraffin-embedded (FFPE) sections were incubated with anti-CD44 antibody (clone 156-3C11; Cell Signaling, Danvers, MA, USA) followed by Histofine Simple Stain MAX PO (Nichirei, Tokyo, JAPAN). A DAB kit (VECTOR, Burlingame, CA, USA) was used to develop the signals. Counterstaining was performed with hematoxylin.

Gene expression analysis. Total RNA was isolated with an RNeasy plus mini kit (Qiagen, Hilden, NRW, Germany). RNA concentration and quality were measured using a NanoDrop ND-1000 spectrophotometer (Nano-Drop Technologies, Wilmington, DE). cDNA was synthesized with a High Capacity RNA-to-cDNA Kit (Applied Biosystems, Thermo Fisher Scientific). TaqMan Gene Expression Master Mix (Applied Biosystems) and the following TaqMan probes (Applied Biosystems) were used for quantitative PCR: CD44 (Hs01075864_m1), CXCR4 (Hs00607978_s1), Nanog (Hs02387400_g1), ALDH1A1 (Hs00946916_m1), ALDH3A1 (Hs00964880_m1) and WNT5A (Hs00998537_m1), while ACTB (Hs99999903_m1) was used as reference gene. Real-time PCR reactions were conducted with a QuantStudio 3 Real Time PCR System or ABI PRISM 7000 Sequence Detection System (Applied Biosystems). For microarray analysis, SurePrint G3 Human GE microarray 8x60K Ver. 3.0 (Agilent Technologies, Santa Clara, CA, USA) was used.

Chemosensitivity analysis. COLO 201 cells $\left(6 \times 10^{3}\right.$ cells/well) were exposed to titrated concentrations of 5-FU in 96-well plates. After 4 days, the percentages of viable cells were determined by WST- 8 assay (Kishida Chemical, Osaka, Japan) according to the manufacturer's instructions. For apoptosis detection, COLO 201 cells $\left(8 \times 10^{4}\right.$ cells/well $)$ were cultured on a 24 -well plate in the presence of a different concentrations $(0.2 \mu \mathrm{M}, 2 \mu \mathrm{M}$, and $20 \mu \mathrm{M})$ of 5-FU. After 4 days, the cells were stained with PE-labeled antiCD44 antibody and examined with an AnnexinV Apoptosis Detection Kit APC (eBioscience, Thermo Fisher Scientific) according to the manufacturer's instructions.

Statistical analysis. Statistical analysis was performed using GraphPad Prism 8 software (GraphPad Software, Inc). Results are presented as mean $\pm \mathrm{SD}$ of three independent experiments. The significance of differences among groups was determined by the Student's $t$-test. Statistical significance was considered as $p<0.05$. The results are marked as $* p<0.05, * * p<0.01$ and $* * * p<0.001$.

\section{Results}

Three cell lines had two populations differing in the expression of potential CSC markers. In order to identify CRC cell lines that include putative CSCs and other cells, we searched for previously reported CSC markers for human CRC (CD44, CD133 and CD166) on the cell surface in those cell lines. These markers were heterogeneously expressed among the different cell lines (Figure 1). CD44 was expressed on all NCI-H747, SW48, WiDr, LS 180, LS411N and HT-29 cells, but only on a restricted population of the COLO 201, COLO 320DM and HCT-15 cell lines. CD44 ${ }^{+}$cells accounted for $33.8 \%, 88.5 \%$ and $27.7 \%$, respectively, of COLO 201, COLO 320DM and HCT-15. CD133 was expressed by most WiDr, SW48, LS411N, HT-29, and COLO 201 cells, but not by the NCI-H747, COLO 320DM, and HCT-15 cell lines. CD166 was expressed by most cells in all cell lines. We decided to focus on three CRC cell lines, HCT-15, COLO 320DM and COLO 201, to identify putative CSCs.

$\mathrm{CD} 4^{+} \mathrm{COLO} 201$ cells showed higher tumor formation capacity than their counterparts in immunodeficient mice. We evaluated the tumorigenic capacity of putative CSC populations in immunodeficient mice. CD44 ${ }^{+}$COLO 201 led to increased tumor development than $\mathrm{CD}_{4} 4^{-} \mathrm{COLO} 201$ $(p<0.001)$, but there were no differences between $\mathrm{CD} 44^{+}$ cells and CD44- cells in HCT-15 and COLO 320DM cell lines (Figure 2). To further assess the tumorigenic capacity of CD44 ${ }^{+}$COLO 201 cells, CD44 ${ }^{+}$and $\mathrm{CD} 44^{-}$cells were injected into NOD/SCID mice with monitoring until 49 days post injection. A total of $3 \times 10^{3}$ of $\mathrm{CD} 44^{+}$cells formed 
$\mathrm{NCl}-\mathrm{H} 747$

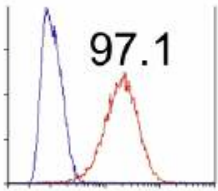

SW48

WiDr

LS 180

LS411N
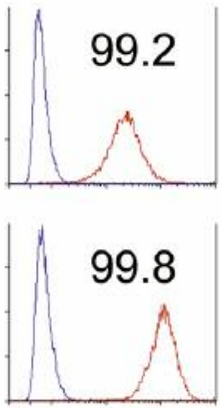

99.7
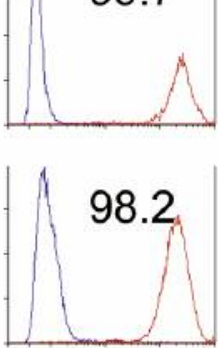

HT-29

COLO 201

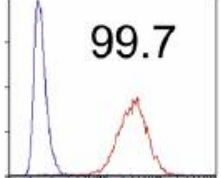

33.8
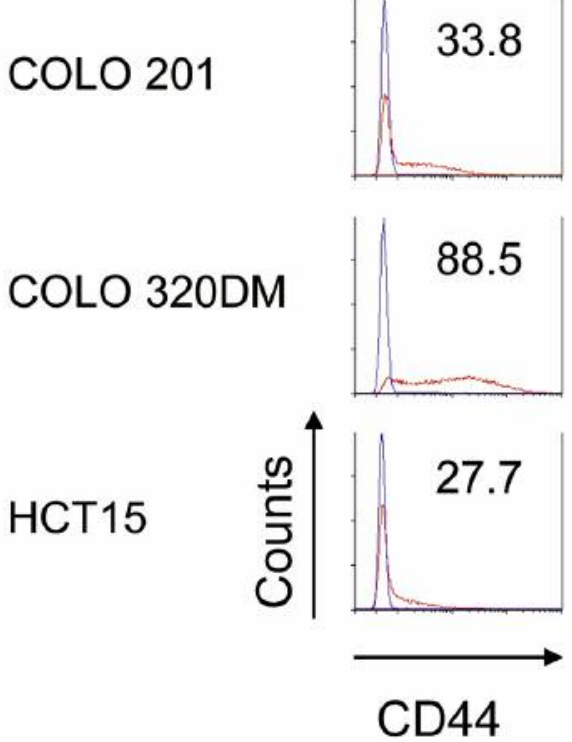
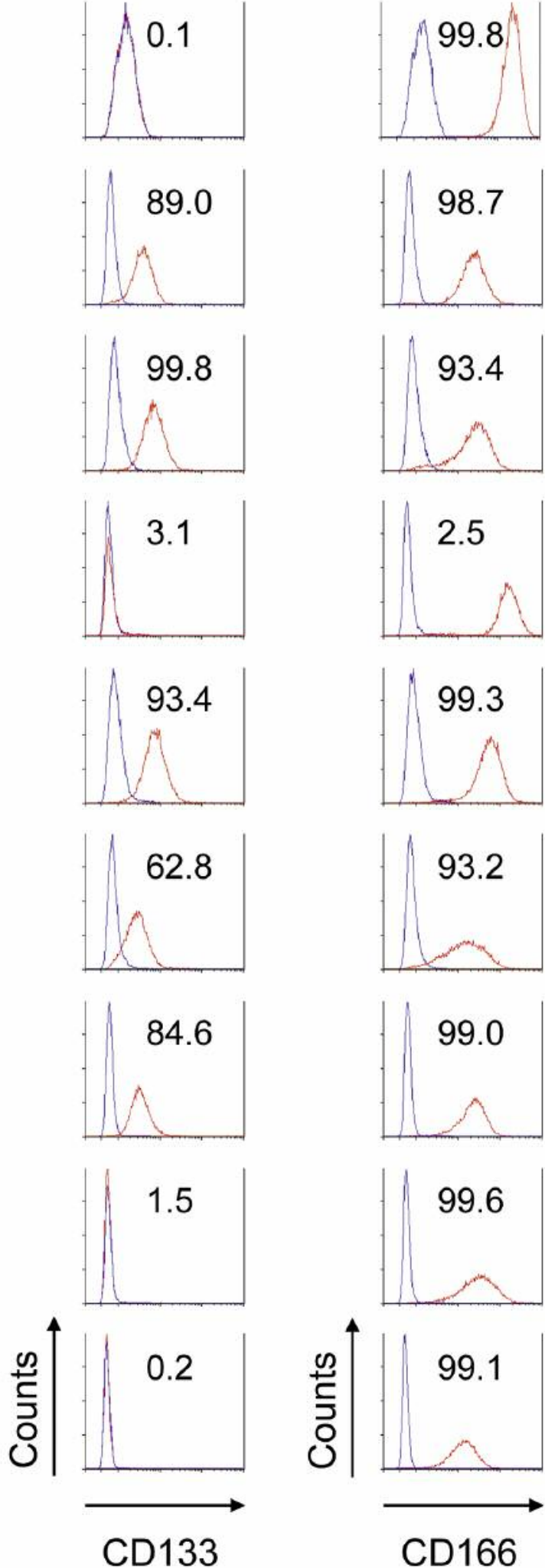

Figure 1. Three colorectal cancer cell (CRC) lines had a CD44+ population. Nine CRC cell lines were analyzed for cell surface expression of CD44, CD133, and CD166 using flow cytometry. Red histograms represent the isotype control-stained cells and blue histograms represent the antibodystained cells. 
tumors with the probability of three out of ten, whereas $\mathrm{CD}_{4} 4^{-}$cells did not form tumors even at $3 \times 10^{4}$ cells (Figure 2B). Thus, we chose COLO 201 as a CSC model and evaluated their CSC properties.

CD $44^{+}$COLO 201 cells were found to possess stemness properties. A sphere culture system is commonly used to identify stemness of normal and cancer cells (12). When parental COLO 201 cells were cultured in a sphere culture medium for 2 weeks, they became highly enriched with CD44 ${ }^{+}$ cells (23.5\% to $83.1 \%$ ) (Figure 3A). Nanog has been reported as the key transcription factor that regulates both self-renewal and pluripotency of cancer cells (13). Nanog expression after sphere culture was also significantly higher $(p<0.001)$ than that in normal culture (Figure 3B). These results suggested that $\mathrm{CD} 44^{+}$cells possess stemness properties. Next, to compare the self-renewal and differentiation potency of the $\mathrm{CD} 44^{+}$and CD $44^{-}$cells, sorted single $\mathrm{CD} 44^{+}$and $\mathrm{CD} 44^{-}$cells were plated in normal growth media in each well of a 96-well plate for 3 weeks. CD $44^{-}$cells only generated CD $44^{+}$cells in three out of nine wells, but $\mathrm{CD} 44^{+}$cells generated $\mathrm{CD} 44^{-}$and $\mathrm{CD} 44^{+}$cells in all nine wells (Figure 3C). Moreover, tumors formed from CD $44^{+}$cells included both $\mathrm{CD} 44^{+}$and $\mathrm{CD} 44^{-}$cells (Figure 3D). This finding clearly indicated that $\mathrm{CD} 44^{+}$cells have the potential for self-renewal and differentiation.

$\mathrm{CD}_{4} 4^{+} \mathrm{COLO} 201$ cells displayed chemoresistance to 5-FU treatment. COLO 201 cells were exposed to 5 -FU to define the dose-response curve (Figure 4A) Next, the proportion of $\mathrm{CD} 44^{+}$and $\mathrm{CD}_{4} 4^{-}$cells was analyzed after 4 days exposure to different concentrations of 5 -FU. The $\mathrm{CD} 44^{+}$population significantly increased in a concentration-dependent manner (Figure 4B). In order to compare the sensitivities of CD44 ${ }^{+}$and $\mathrm{CD} 44^{-}$cells to 5-FU, sorted cells were cultured in the presence of $200 \mathrm{nM}$ of 5-FU for 4 days. The apoptotic and/or necrotic proportions of $\mathrm{CD} 44^{+}$cells were significantly lower $(p<0.01)$ than those of $\mathrm{CD}_{4} 4^{-}$cells (Figure $4 \mathrm{C}$ ). These results indicated that the $\mathrm{CD} 44^{+}$population was resistant to 5 -FU treatment.

Cancer stemness-related genes were up-regulated in $\mathrm{CD}_{4} 4^{+}$ COLO 201 cells. To further clarify the CSC-like properties of CD $44^{+}$COLO 201 cells, we performed DNA microarray analysis for systematic gene expression profiling of $\mathrm{CD} 44^{+}$and $\mathrm{CD}_{4} 4^{-}$cells. Based on the criteria of a fold change of $\geq 2$ and a $p$-value ( $t$-test) of $<0.05,407$ genes were extracted as differentially expressed, including 191 up-regulated and 216 down-regulated genes (Figure 5A). The expression levels of genes related to cancer stemness and chemoresistance, such as CXCR4, ALDH1A1, ALDH3A1 and WNT5A, were high in $\mathrm{CD} 44^{+}$cells; these genes were further verified using quantitative real-time PCR (Figure 5B). These data demonstrated that CD44 ${ }^{+}$ COLO 201 cells possess many CSC features and could be used as a model to accelerate CSC research.
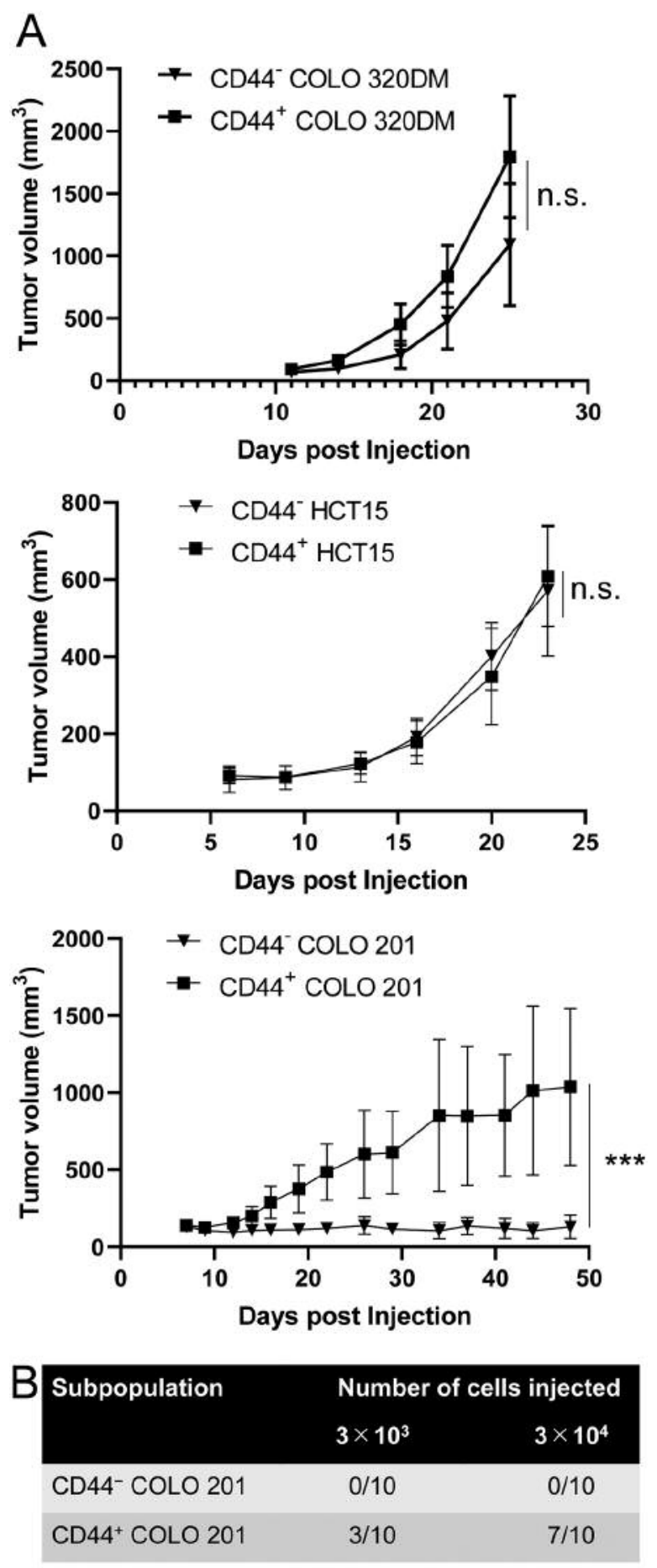

Figure 2. $\mathrm{CD}_{4} 4^{+} \mathrm{COLO} 201$ cells displayed higher tumor formation capacity in immunodeficient mice than CD44- COLO 201 cells. We evaluated the tumor formation capacities of $\mathrm{CD}_{4} 4^{+}$and $\mathrm{CD}_{4} 4^{-}$cells in three CRC lines. HCT15, COLO 320DM, and COLO 201 were injected at $2 \times 10^{4}, 3 \times 10^{4}$ and $5 \times 10^{4}$ into nude mice, respectively. Data are presented as mean $\pm S D(A)$. Tumor incidence of $C D 44^{+}$and $C D 44^{-}$ COLO 201 cells. The cell numbers indicated in the figure were injected into NOD/SCID mice with monitoring for 49 days post injection. Data represent the number of tumors formed/number of injections $(B)$. 
A

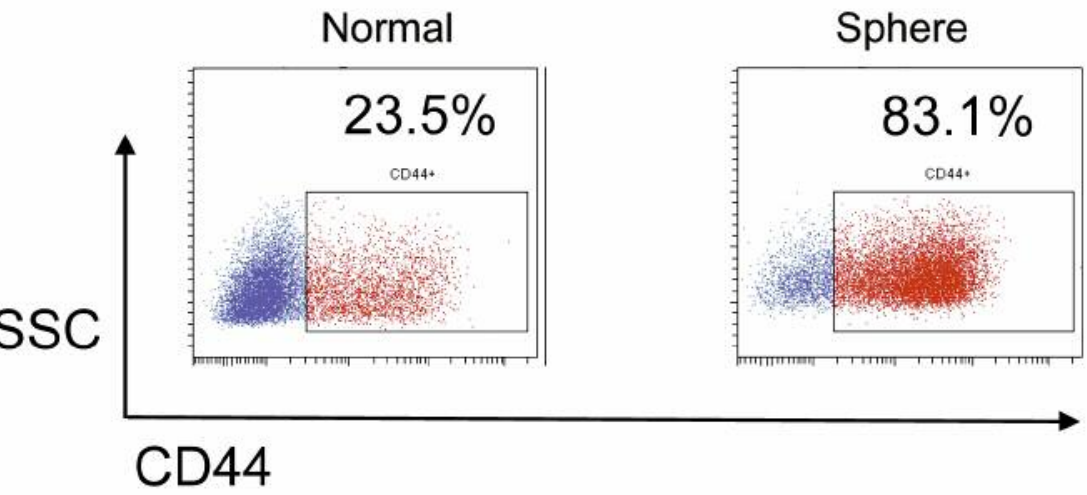

$\mathrm{B}$

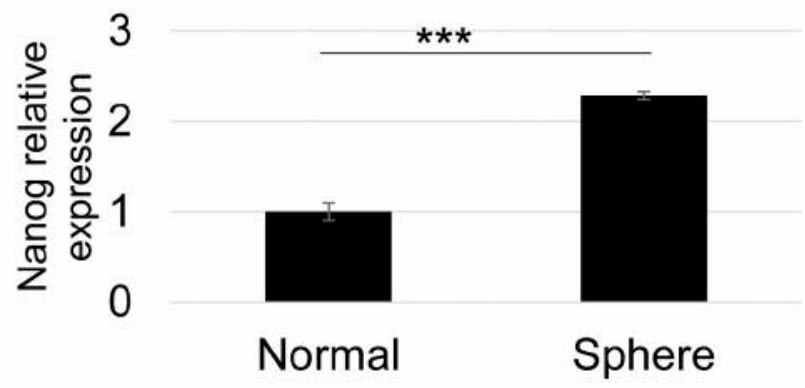

C

CD44-

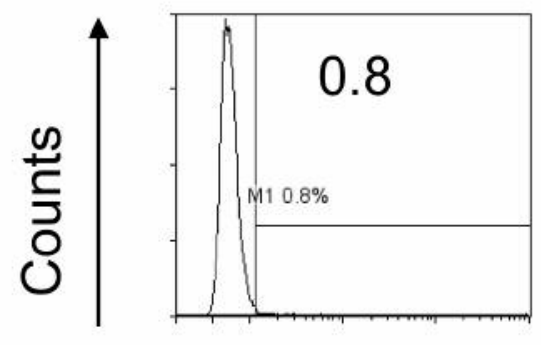

CD44+

\section{CD44}
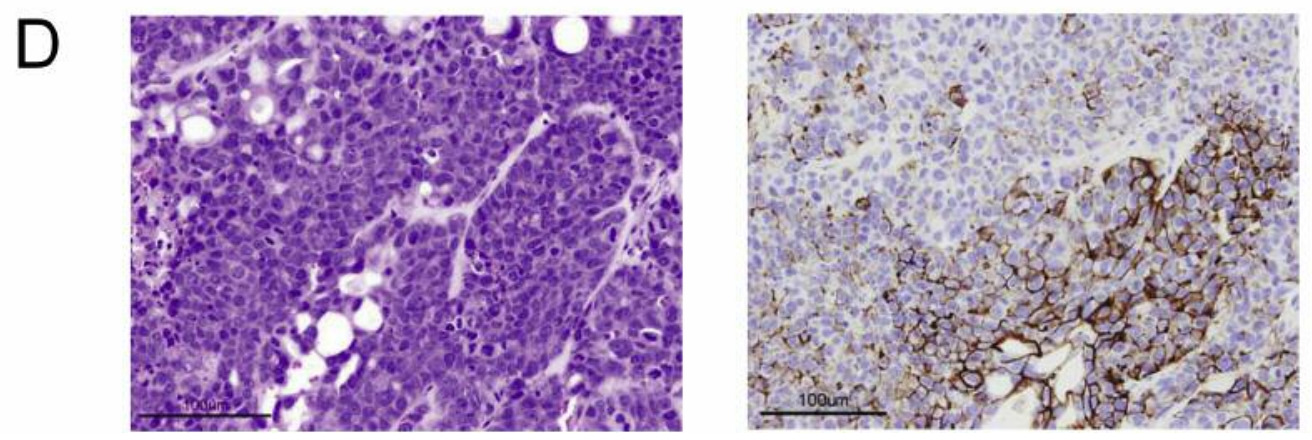

Figure 3. $\mathrm{CD}_{4} 4^{+} \mathrm{COLO} 201$ cells possessed more stemness properties. Parental COLO 201 cells were cultured in sphere-forming medium for 2 weeks and then analyzed using flow cytometry $(A)$ and quantitative real-time PCR (B). The percentage of $C D 44^{+}$cells in each well was analyzed using flow cytometry. Representative data are shown. Graphs represent the mean $\pm S D$ of three independent experiments $(C)$. Histopathology (hematoxylin \& eosin staining) and CD44 expression of tumor tissues in nude mice. Tumor tissues were obtained 21 days after injection of CD44+ cells $(D)$. 
A

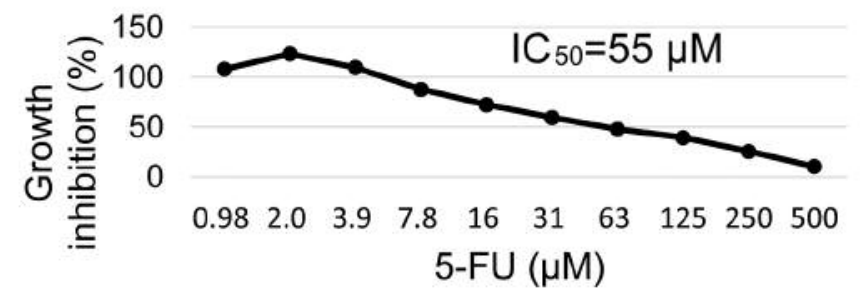

B
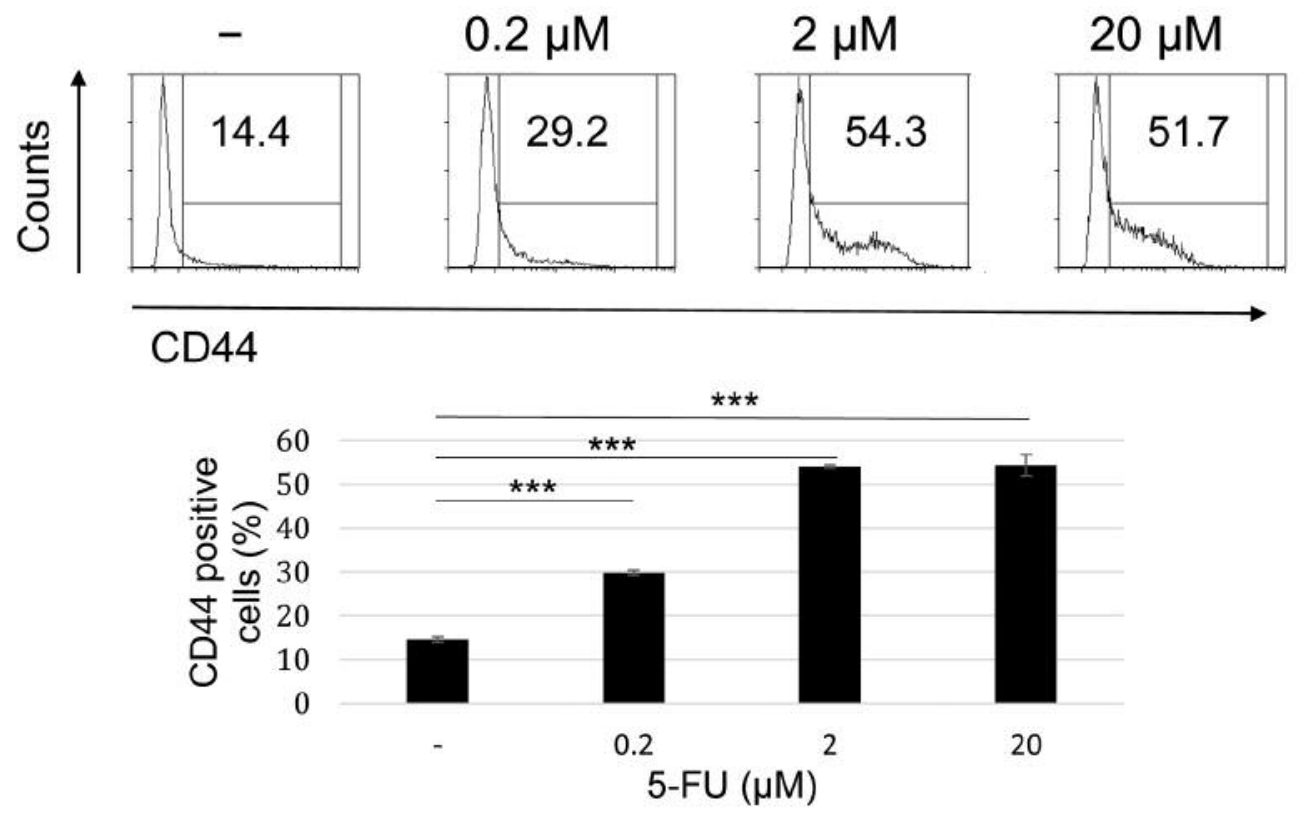

C
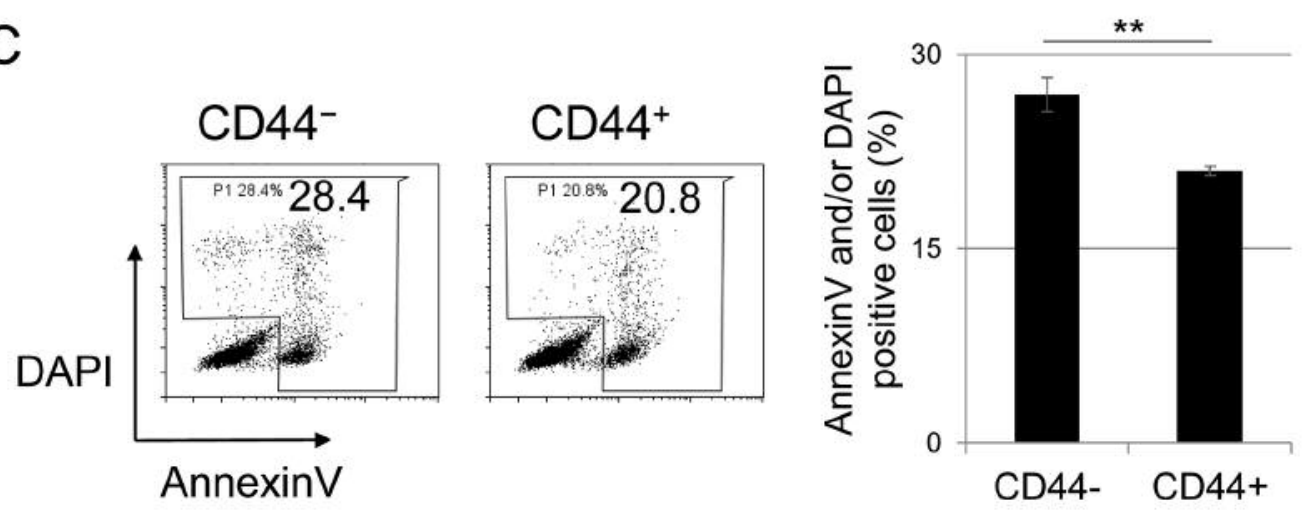

Figure 4. $\mathrm{CD}_{4} 4^{+} \mathrm{COLO} 201$ cells displayed chemoresistance to 5-FU treatment. The dose response curve of 5-FU in COLO 201. Cells were cultured with titrated doses of 5-FU for 4 days, and the number of live cells was obtained by the WST-8 cell viability assay (A). COLO 201 cells were cultured with the indicated doses of 5-FU for 4 days and, the percentage of $C D 44^{+}$cells was analyzed using flow cytometry $(B)$. CD44+ or CD44cells sorted from COLO 201 were cultured with $200 \mathrm{nM}$ 5-FU for 4 days. Cells stained with AnnexinV and DAPI were measured using flow cytometry (C). Each bar represents mean \pm SD of three independent experiments.

\section{Discussion}

Our data show that COLO 201 cells can be separated into two populations based on CD44 expression. COLO 201 cells in this study consisted of $10-35 \% \mathrm{CD}_{4} 4^{+}$cells, while in literature COLO 201 cells have been reported to only include $0.5 \% \mathrm{CD}_{4} 4^{+}$cells (14). Our cells were purchased from ATCC and were certificated as COLO 201 cell line after the investigation of a profile of short tandem repeats. CD44 protein is reported to be controlled at multiple steps, 
A

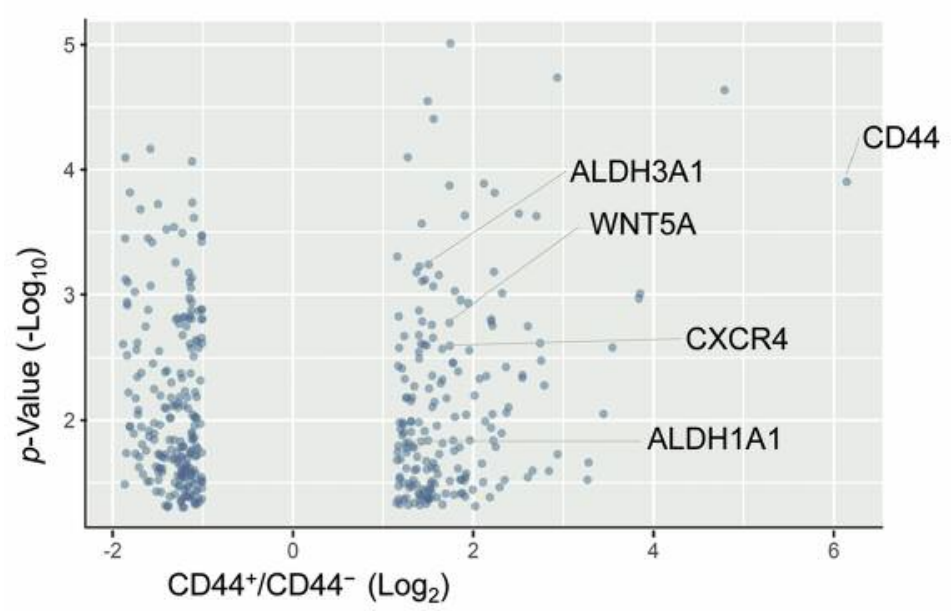

B

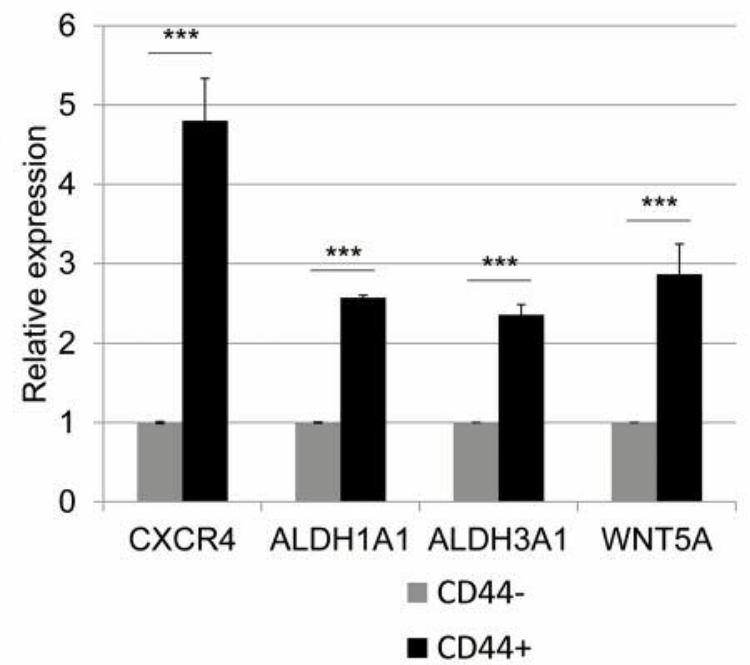

Figure 5. Cancer stemness and chemoresistance-related genes were up-regulated in CD44+ COLO 201 cells. Microarrays were used to analyze comprehensive gene expression in $\mathrm{CD}_{4} 4^{+}$and $\mathrm{CD} 44^{-}$sorted cells from COLO 201 cells. The gene plot shows 191 up-regulated genes and 206 down-regulated genes in $\mathrm{CD}_{4}{ }^{+} \mathrm{COLO} 201$ cells. The data obtained from three independent experiments (A). The expressions of the mRNAs encoding CXCR4, ALDH1A1, ALDH3A1 and WNT5A were analyzed in $\mathrm{CD}_{4} 4^{+}$and CD44- COLO 201 cells using quantitative real-time PCR (B). Graphs represent the mean $\pm S D$ of three independent experiments.

including transcriptional regulation, alternative splicing, and posttranslational modifications (15). It has been reported that cell passaging and cell culture conditions affect the gene expression and the differentiation status of cells (16). In the previous study (14), nutrient-rich culture condition was applied, which was not the case in the present one; thus, this fact could explain the different $\mathrm{CD} 44^{+}$cell ratio between the two different studies.

When the sorted CD $44^{+}$or CD44- COLO 201 cells were transplanted into mice, $\mathrm{CD} 44^{+}$cells induced larger tumor volume and a greater tumor formation capacity in vivo compared to $\mathrm{CD}_{4} 4^{-}$cells (Figure 2A, B). These results suggested that $\mathrm{CD} 44^{+}$COLO 201 cells might have CSC properties. Then, we confirmed that parental COLO 201 cells became highly enriched with $\mathrm{CD} 44^{+}$cells in sphere culture, and the expression level of Nanog was up-regulated compared to cells in normal culture (Figure 3A, B). Moreover, CD44 ${ }^{+}$ cells, which could also generate CD $44^{-}$cells, possessed higher differentiation potency in vivo and in vitro (Figure $3 \mathrm{C}, \mathrm{D})$. In addition, $\mathrm{CD} 44^{+}$cells displayed higher resistance to 5 -FU treatment compared to $\mathrm{CD}^{-} 4^{-}$cells (Figure 4). Therefore, these results indicate that $\mathrm{CD} 44^{+}$cells found in COLO 201 have high tumorigenic potential, stem cell marker expression, differentiation potency and chemoresistance, features that characterize primary CSCs in CRC (17).

This study revealed that CD44 could be a reliable CSC marker in CRCs, while it has been reported that combining multiple markers with CD44 enables identification of a more specific CSC population (6). However, CD $44^{+}$cells were not separated into subpopulations based on previously reported CSC surface markers (CD133, CD166) expressed by CRCs (Figure 1). Microarray analysis identified that $C X C R 4$, $A L D H 1 A 1, A L D H 3 A 1$ and WNT5A genes, which have been reported as contributing to cancer stemness and chemoresistance (18-21), were up-regulated in $\mathrm{CD}_{4} 4^{+}$cells . Therefore, combining these molecules as markers with CD44 could identify CSC population more specifically.

The CSC model reported here shows a clear difference in tumorigenicity compared to other previously reported cell lines $(22,23)$; moreover, CD $44^{+}$COLO 201 cells possess many CSC features. Therefore, our results suggest that the present CSC model could be a valuable tool to accelerate CSC research. Since significant differences in gene expression between CD $44^{+}$ and $\mathrm{CD}_{4} 4^{-}$cells were only noted for 407 genes (Figure 5A), further research may reveal novel targets against CSCs.

\section{Conflicts of Interest}

The Authors confirm that there are no conflicts of interest in regard to this study.

\section{Authors' Contributions}

Study concept and design: H. Okuyama, W. Nogami, Y. Sato; Animal study: H. Okuyama, W. Nogami, H. Yoshida; Experiments: H. Okuyama, W. Nogami, Y. Sato; Analysis and interpretation of data: H. 
Okuyama, W. Nogami, Y. Sato, Y. Tona; Drafting of the manuscript: H. Okuyama, Y. Sato, W. Nogami, Y. Tona; Study supervision: Y. Tanaka.

\section{Acknowledgements}

The Authors thank Takeshi Akahane who provided immunohistochemistry services on behalf of the Applied Medical Research Laboratory. We thank Tetsuya Yoshida for assistance with the data analysis.

\section{References}

1 Bray F, Ferlay J, Soerjomataram I, Siegel RL, Torre LA and Jemal A: Global Cancer Statistics 2018: GLOBOCAN Estimates of incidence and mortality worldwide for 36 cancers in 185 countries. CA Cancer J Clin 68: 394-424, 2018. PMID: 30207593. DOI: $10.3322 /$ caac. 21492

2 Reya T, Morrison SJ, Clarke MF and Weissman IL: Stem cells, cancer, and cancer stem cells. Nature 414(6859): 105-111, 2001. PMID: 11689955 . DOI: $10.1038 / 35102167$

3 Bao S, Wu Q, McLendon RE, Hao Y, Shi Q, Hjelmeland AB, Dewhirst MW, Bigner DD and Rich JN: Glioma stem cells promote radioresistance by preferential activation of the DNA damage response. Nature 444(7120): 756-760, 2006. PMID: 17051156. DOI: 10.1038 /nature05236

4 Keshet GI, Goldstein I, Itzhaki O, Cesarkas K, Shenhav L, Yakirevitch A, Treves AJ, Schachter J, Amariglio N and Rechavi G: MDR1 expression identifies human melanoma stem cells. Biochem Biophys Res Commun 368(4): 930-936, 2008. PMID: 18279661. DOI: 10.1016/j.bbrc.2008.02.022

5 Yang T, Rycaj K, Liu ZM and Tang DG: Cancer stem cells: constantly evolving and functionally heterogeneous therapeutic targets. Cancer Res 74(11): 2922-2927, 2014. PMID: 24713433. DOI: 10.1158/0008-5472.CAN-14-0266

6 Taniguchi H, Suzuki Y and Natori Y: The evolving landscape of cancer stem cells and ways to overcome cancer heterogeneity. Cancers (Basel) 11(4): E532, 2019. PMID: 31013960. DOI: 10.3390/cancers 11040532

7 Dalerba P, Dylla SJ, Park IK, Liu R, Wang X, Cho RW, Hoey T, Gurney A, Huang EH, Simeone DM, Shelton AA, Parmiani G, Castelli $\mathrm{C}$ and Clarke MF: Phenotypic characterization of human colorectal cancer stem cells. Proc Natl Acad Sci USA 104(24): 1015810163, 2007. PMID: 17548814. DOI: 10.1073/pnas.0703478104

8 Ricci-Vitiani L, Lombardi DG, Pilozzi E, Biffoni M, Todaro M, Peschle $\mathrm{C}$ and De Maria R: Identification and expansion of human colon-cancer-initiating cells. Nature 445(7123): 111-115, 2006. PMID: 17122771. DOI: $10.1038 /$ nature05384

9 Barker N, van Es JH, Kuipers J, Kujala P, van den Born M, Cozijnsen M, Haegebarth A, Korving J, Begthel H, Peters PJ and Clevers H: Identification of stem cells in small intestine and colon by marker gene Lgr5. Nature 449: 1003-1007, 2007. PMID: 17934449. DOI: 10.1038/nature06196

10 Desai A, Yan Y and Gerson SL: Concise reviews: Cancer stem cell targeted therapies: toward clinical success. Stem Cells Transl Med 8(1): 75-81, 2019. PMID: 30328686. DOI: 10.1002/sctm. 18-0123

11 Jordan CT: Cancer stem cells: controversial or just misunderstood? Cell Stem Cell 4(3): 203-205, 2009. PMID: 19265659. DOI: 10.1016/j.stem.2009.02.003
12 Weiswald LB, Bellet D and Dangles-Marie V: Spherical cancer models in tumor biology. Neoplasia 17(1): 1-15, 2015. PMID: 25622895. DOI: 10.1016/j.neo.2014.12.004

13 Jeter CR, Yang T, Wang J, Chao HP and Tang DG: Concise review: NANOG in cancer stem cells and tumor development: an update and outstanding questions. Stem Cells 33(8): 23812390, 2015. PMID: 25821200. DOI: 10.1002/stem.2007

14 Muraro MG, Mele V, Däster S, Han J, Heberer M, Cesare Spagnoli G and Iezzi G: $\mathrm{CD} 133^{+}, \mathrm{CD} 166^{+} \mathrm{CD} 44^{+}$, and $\mathrm{CD} 24^{+} \mathrm{CD} 44^{+}$phenotypes fail to reliably identify cell populations with cancer stem cell functional features in established human colorectal cancer cell lines. Stem Cells Transl Med 1(8): 592-603, 2012. PMID: 23197865. DOI: 10.5966/sctm.2012-0003

15 Chen C, Zhao S, Karnad A and Freeman JW: The biology and role of CD44 in cancer progression: therapeutic implications. J Hematol Oncol 11(1): 64, 2008. PMID: 29747682. DOI: 10.1186/s13045-018-0605-5

16 Hughes P, Marshall D, Reid Y, Parkes H and Gelber C: The costs of using unauthenticated, over-passaged cell lines: how much more data do we need? Biotechniques 43(5): 575-586, 2007. PMID: 18072586 . DOI: $10.2144 / 000112598$

17 Munro MJ, Wickremesekera SK, Peng L, Tan ST and Itinteang T: Cancer stem cells in colorectal cancer: a review. J Clin Pathol 71(2): 110-116, 2018. PMID: 28942428. DOI: $10.1136 /$ jclinpath2017-204739

18 Domanska UM, Kruizinga RC, Nagengast WB, Timmer-Bosscha $\mathrm{H}$, Huls G, de Vries EG and Walenkamp AM: A review on CXCR4/CXCL12 axis in oncology: no place to hide. Eur J Cancer 49(1): 219-230, 2013. PMID: 22683307. DOI: 10.1016/ j.ejca.2012.05.005

19 Januchowski R, Wojtowicz K and Zabel M: The role of aldehyde dehydrogenase (ALDH) in cancer drug resistance. Biomed Pharmacother 67(7): 669-680, 2013. PMID: 23721823. DOI: 10.1016/j.biopha.2013.04.005

20 Asem MS, Buechler S, Wates RB, Miller DL and Stack MS: Wnt5a signaling in cancer. Cancers (Basel) 8(9): 79, 2016. PMID: 27571105. DOI: 10.3390/cancers8090079

21 Chatterjee S, Behnam Azad B and Nimmagadda S: The intricate role of CXCR4 in cancer. Adv Cancer Res 124: 31-82, 2014. PMID: 25287686. DOI: 10.1016/B978-0-12-411638-2.00002-1

22 Zhou JY, Chen M, Ma L, Wang X, Chen YG and Liu SL: Role of CD44(high)/CD133(high) HCT-116 cells in the tumorigenesis of colon cancer. Oncotarget 7(7): 7657-7666, 2016. PMID: 26840024. DOI: 10.18632 /oncotarget.7084

23 Munakata K, Uemura M, Tanaka S, Kawai K, Kitahara T, Miyo M, Kano Y, Nishikawa S, Fukusumi T, Takahashi Y, Hata T, Nishimura J, Takemasa I, Mizushima T, Ikenaga M, Kato T, Murata K, Carethers JM, Yamamoto H, Doki Y and Mori M: Cancer stem-like properties in colorectal cancer cells with low proteasome activity. Clin Cancer Res 22(21): 5277-5286, 2016. PMID: 27166395. DOI: 10.1158/1078-0432.CCR-15-1945 